PS3-24:

\section{HMORN Sites: Organization Models and Their Data}

Amy Butani ${ }^{1}$

${ }^{1}$ HealthPartners

Background/Aims: We aim to describe the various organization models across the HMORN and the resulting data. Methods: Each HMORN site has patients and/or insurance members. The data available for these two populations are different; the differences will be demonstrated. Results: The VDW has been designed to accommodate data for both member and patient populations. Conclusions: Understanding the data available for each population will help in designing studies, especially multi-site studies. Keywords: HMO Model; Data; VDW

doi:10.3121/cmr.2013.1176.ps3-24

PS3-27:

Virtual Tumor Registry or Albatross

Rick Krajenta ${ }^{1}$

${ }^{1}$ Henry Ford Health System / Health Alliance Plan

Background/Aims: Creating a virtual tumor registry to function within the Cancer Research Network (CRN) enables us to identify tumors in our members. This is critical for performing cancer research for the CRN. Data standards and staging schema are defined by regulating agencies external to $\mathrm{CRN}$ and require all users to adhere to these standards. This establishes a gold standard for modeling our data structure that all participating plan sites should be able to achieve. Methods: Initial datasets were modeled from a commercial registry package. The vendor-provided data dictionary included variable names, formats, and length. The data dictionary also included whether the variable was required or optional, and if the data entry was system-provided or added with keyboard entry. Unfortunately, health planspecific data characteristics were soon added and the virtual tumor registry began to diverge from the gold standard. It had been assumed that it was a static target (i.e., not requiring adjustment). However, the regulating agencies had changed the standards and a major revision was impending. To remain compliant with the gold standard, the tumor registry would need to be expanded to 117 variables and the data needs to be destroyed and rebuilt each time the data is refreshed. Results: Once expanded to 117 variables, we are having difficulties getting health plans re-synchronized with the data standards. We have developed some QA SAS code to investigate anomalous findings and return results to the health plan. Conclusions: The virtual tumor registry is the key dataset to allow the $\mathrm{CRN}$ to function as a cancer research consortium. It allows the identification of tumor diagnoses, tumor staging, initial cancer directed treatment and annual follow-up. Recent additions capture advanced testing including immunostains, nucleic acid polymorphisms or mutations, and other personalized prognostic molecular markers. The virtual tumor registry can be used for cancer research feasibility estimates, treatment modality identification, survival calculations and molecular marker studies.

Keywords: Tumor Registry; Database

doi:10.3121/cmr.2013.1176.ps3-27

PS3-28:

The Evolution of Tumor Standards and Its Effect on Observational Data

Jack Richter $^{1}$; Lela McFarland ${ }^{1}$; Chu-Ling $\mathrm{Yu}^{1}$

${ }^{1}$ Kaiser Permanente Mid-Atlantic

Background/Aims: Quality information is essential to analyzing tumor data. Tumor data standards change over time, making it paramount to understand how the external standards affect the data. The changes, over time, can create artificial data quality issues that result in inaccurate conclusions and inhibit observational research. Improving the quality of data, information, and knowledge is foundational to utilizing the VDW Tumor data for cancer research. The challenges associated with maintaining high quality data in the VDW Tumor data requires a clear understanding of the various data content standards that have historically affected these data. As experts on their tumor data, tumor researchers need to understand and identify which data content standards affect their data. They also need to know how the data standards were implemented and managed in their systems as well as in the standards world of tumor data. Methods: We gathered information from the tumor standards agencies and compiled into one document. We then evaluated each change in the standards for its potential impact on VDW Tumor data elements. Results: We developed a timeline/calendar view to show the version changes implemented, by date for each standard. For each version change, a callout contains a table with the VDW Tumor data elements that were impacted and a description of the impact. Conclusions: VDW Tumor data content is impacted by various standards, and when utilizing and loading this data, they must be taken into account. This presentation provides a guideline of the changes over time that can be used when accessing or loading VDW Tumor data.

Keywords: Tumor; Data; VDW

doi:10.3121/cmr.2013.1176.ps3-28 